\title{
Importance of Facial Plastic Surgery Education in Residency: A Resident Survey
}

\author{
Steven A. Curti ${ }^{1}$ J. Randall Jordan ${ }^{1}$ \\ ${ }^{1}$ Department of Otolaryngology, University of Mississippi Medical \\ Center, Jackson, Mississippi, United States \\ Int Arch Otorhinolaryngol 2020;24(3):e278-e281.
}

\begin{abstract}
Address for correspondence Steven A. Curti, MD, Department of Otolaryngology, University of Mississippi Medical Center, 2500 North State Street, Jackson, MS 39216, United States (e-mail: scurti@umc.edu).
\end{abstract}

\begin{abstract}
Keywords

- facial plastic surgery

- residency

- survey

- education

Introduction Facial plastic and reconstructive surgery (FPRS) is a key part of the curriculum for otolaryngology residents. It is important to gain an understanding of the breadth of exposure and level of competence residents feel with these concepts during their residency.

Objective To determine the level of FPRS exposure and training otolaryngology residents receive during their residency.

Methods A survey was emailed to all Accreditation Council for Graduate Medical Education (ACGME) accredited otolaryngology residents. The survey aimed to find the level of exposure to FPRS procedures otolaryngology residents get and how confident they feel with their training in cosmetic FPRS.

Results A total of 213 residents responded to the survey for an overall response rate of $13.4 \%$. There was an even mixture of residents from all postgraduate year (PGY) levels, with $58 \%$ of respondents being male. Almost all (98\%) of the residents felt FPRS was important to otolaryngology residency training. Exposure to procedures varied with $57 \%$ performing or assisting with cosmetic minor procedures, $81 \%$ performing or assisting with cosmetic major procedures, and $93 \%$ performing or assisting with reconstructive procedures. Only $49 \%$ of residents felt their programs either very or somewhat adequately prepared them in cosmetic facial plastic surgery.

Conclusion There was a wide variability in the FPRS procedure exposure. Most residents felt procedures were a vital part of otolaryngology residency training, but not all were able to participate in them. Only half of the residents felt well-prepared in cosmetic procedures.
\end{abstract}

\section{Introduction}

Facial plastic and reconstructive surgery (FPRS) is a broad field encompassing both aesthetic and reconstructive surgery. All aspects of FPRS are important in the training of otolaryngology residents. These authors have sensed a variation in the level of training for facial plastic and reconstructive surgery, and in particular cosmetic procedures while in residency. In 2017, there were $>17.5$ million cosmetic procedures performed in the United States, a number that continues to grow. There are well-known complications to these procedures and little to no regulation of the practitioners who preform them. ${ }^{1}$ It is apparent that there is a need for well-trained practitioners in cosmetic procedures.

While there is significant overlap in the cosmetic surgery world between different medical specialties, otolaryngologists make up a large portion of specialists who perform facial plastic surgery. ${ }^{1}$ Although a fellowship can improve the knowledge of facial plastic surgery with specific training, the interest in facial plastic surgery procedures continues to rise among received

April 14, 2019

accepted

September 17, 2019

published online

December 13, 2019
DOI https://doi.org/

10.1055/s-0039-1700584. ISSN $1809-9777$.
Copyright (e 2020 by Thieme Revinter

Publicações Ltda, Rio de Janeiro, Brazil
License terms

(요 (1) $\Theta \circledast$ 
practicing otolaryngologists and residents in training. ${ }^{2-5}$ Facial plastic surgery continues to be a mainstay in the core curriculum as specified by the American Board of Otolaryngology and the Accreditation Council for Graduate Medical Education (ACGME) for otolaryngology residency training programs. In the past few decades, there has been a call for an increase in the facial plastic surgery curricula. ${ }^{6-8}$

Our goal was to assess the level of training that otolaryngology residents receive in FPRS, with an emphasis on cosmetic surgery, across the United States. We used a survey, sent out to residents at otolaryngology residency-training programs, to help us assess how residents perceive their training in FPRS.

\section{Methods}

A 15-question survey was created by the authors using Qualtrics (www.qualtrics.com). The survey can be viewed in - Table 1. The survey was sent to all United States Otolaryngology Head and Neck surgery residency program's

Table 1 List of Survey Questions

1. Are you Male or Female?

2. What is your PGY level?

3. Does your residency program have a facial plastic surgery fellowship?

4. How many facial plastic surgery physicians are involved with resident education at your program?

5. Do you have the opportunity to participate in cosmetic minor procedures (injections, lasers, chemical peels, etc.) during your residency training?

6. Which cosmetic minor procedures have you personally administered? (Select all that apply)

7. Do you feel cosmetic minor procedures (injections, lasers, chemical peels, etc.) are a vital part of otolaryngology residency training?

8. Do you have the opportunity to participate in cosmetic major procedures (facelift, rhinoplasty, blepharoplasty, etc.) during your residency training?

9. Do you feel that cosmetic major procedures (facelift, rhinoplasty, blepharoplasty, etc.) are a vital part of otolaryngology residency training?

10. Do you have the opportunity to participate in facial plastic reconstructive procedures (MOHS reconstruction, skin cancer excision, etc.) during your residency training?

11. Do you feel that facial plastic reconstructive procedures (MOHS reconstruction, skin cancer excision, etc.) are a vital part of otolaryngology residency training?

12. Does your program provide you with formal lectures in cosmetic facial plastic surgery topics?

13. Does your training program include a resident run cosmetic clinic?

14. How adequate do you feel that your residency program trains you in cosmetic facial plastic surgery?

15. Are you interested in facial plastic and reconstructive surgery as a fellowship?
Program Directors and Residency Coordinators via list serve email with the assistance of the Otolaryngology Program Directors Organization. The Program Directors and Residency Coordinators were asked to forward to all residents. Two separate reminder emails were subsequently sent out during the three-week period that the survey was open, each one week apart. The survey was open from October 8 , 2018 to October 31, 2018. The study was exempted by the Institutional Review Board. The goal of the survey was to assess resident exposure to different aspects of facial plastic surgery and their comfort with minor and major facial plastic surgery procedures.

\section{Results}

A total of 213 residents responded to the survey for a response rate of $13.38 \%$ (213/1592). The basic demographic information can be viewed in - Table 2.

Over three fourths (82\%) of the residents responded that they feel cosmetic minor procedures (injections, lasers, chemical peels, etc.) are either somewhat or very important as part of otolaryngology residency training; however, $22 \%$ (47/211) of the residents have no exposure to such procedures in their training, and another 21\% (45/211) only have the ability to observe these procedures. Out of the respondents that participate in minor cosmetic procedures, $29 \%$ $(61 / 211)$ serve in the role of assistant surgeon, and $28 \%$

Table 2 Demographics of survey participants

\begin{tabular}{|l|l|}
\hline & $\boldsymbol{n}(\%)$ \\
\hline Total responses & $213(100)$ \\
\hline Gender & \\
\hline Male & $124(58.2)$ \\
\hline Female & $89(41.8)$ \\
\hline PGY level & \\
\hline 1 & $40(18.8)$ \\
\hline 2 & $38(17.8)$ \\
\hline 3 & $42(19.7)$ \\
\hline 4 & $48(22.5)$ \\
\hline 5 & $45(21.1)$ \\
\hline Program have an FP Fellowship? & \\
\hline Yes & $153(71.8)$ \\
\hline No & $60(28.2)$ \\
\hline How many FPRS faculty? & \\
\hline 0 & $11(5.2)$ \\
\hline 1 & $66(31.0)$ \\
\hline 2 & $71(33.3)$ \\
\hline 3 & $36(19.9)$ \\
\hline 4 & $18(84.5)$ \\
\hline 5 or more & $11(5.2)$ \\
\hline
\end{tabular}

Abbreviations: FP, facial plastic; FPRS, facial plastic and reconstructive surgery; PGY, postgraduate year. 


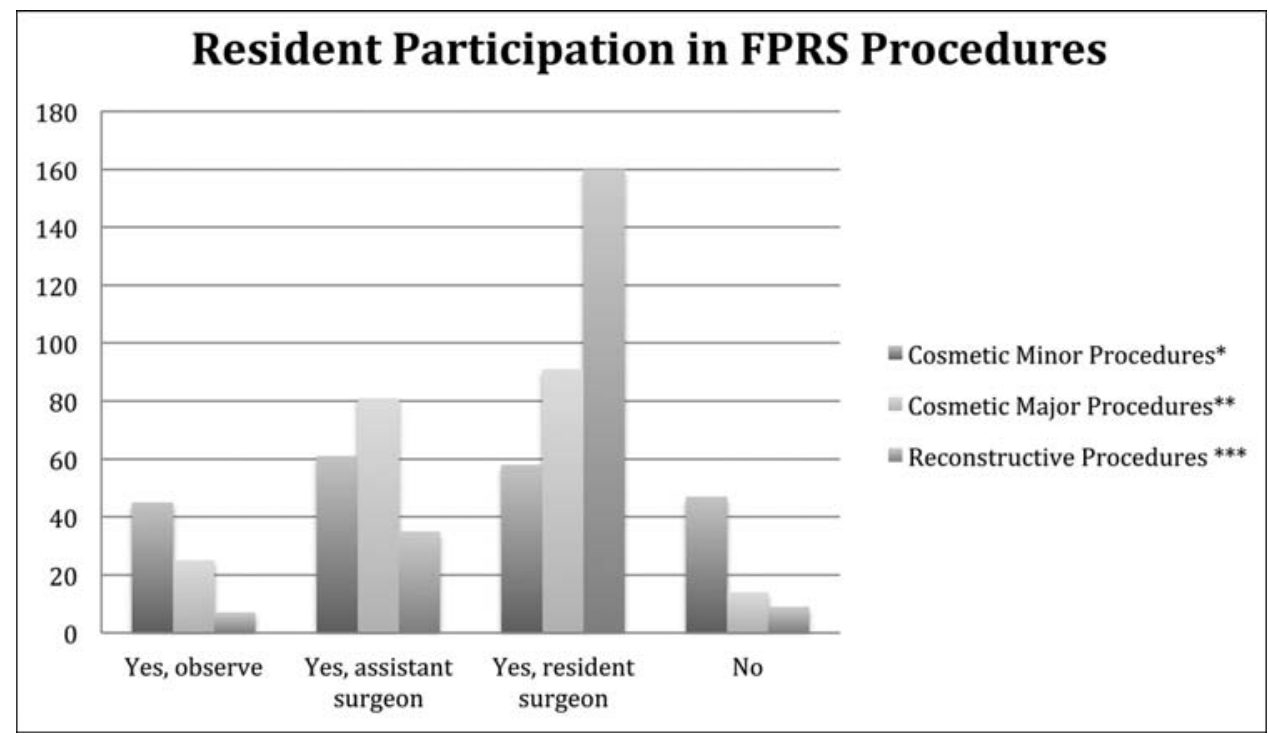

Fig. 1 Resident participation in facial plastic and reconstructive surgery cases. ${ }^{*}$ Minor procedures $=$ (injections, lasers, chemical peels, etc). ${ }^{* *}$ Major procedures $=(\text { facelift, rhinoplasty, blepharoplasty, etc. })^{* * *}$ Reconstructive procedures $=(\mathrm{MOHS}$ reconstruction, skin cancer excision, etc $)$.

(58/211) perform in the role of resident surgeon. Out of these respondents, 37\% have injected neurotoxins (Botox, Dysport, etc.), $25 \%$ have injected dermal soft tissue fillers (Restylane, Juvederm, etc), and $12 \%$ have performed laser skin procedures. Resident participation breakdown can be found in -Fig. 1.

Similarly 93\% (197/211) of the residents responded that they feel major cosmetic procedures (facelift, rhinoplasty, blepharoplasty, etc.) are either somewhat or very important as part of otolaryngology residency training. - Fig. 2 shows how residents responded to the importance of facial plastic and reconstructive cases in their residency training. The majority $(82 \%, 172 / 211)$ of the residents who responded either served in the role of assistant surgeon (38\%) or resident surgeon (44\%). Only two respondents who took the survey did not feel that reconstructive procedures (MOHS reconstruction, skin cancer excision, etc.) were either somewhat or very important as part of otolaryngology residency training. Accordingly, 95\% of the respondents have the opportunity to perform these surgeries in either an assistant or resident surgeon role.

Most residents (86\%) have exposure to formal lectures on facial plastic and reconstructive topics. Only $16 \%$ of the residents have the opportunity to take part in a resident cosmetic clinic. Fewer than half of the residents surveyed $(49 \%, 103 / 210)$ felt that their residency training program either extremely or somewhat adequately trains them in cosmetic facial plastic surgery.

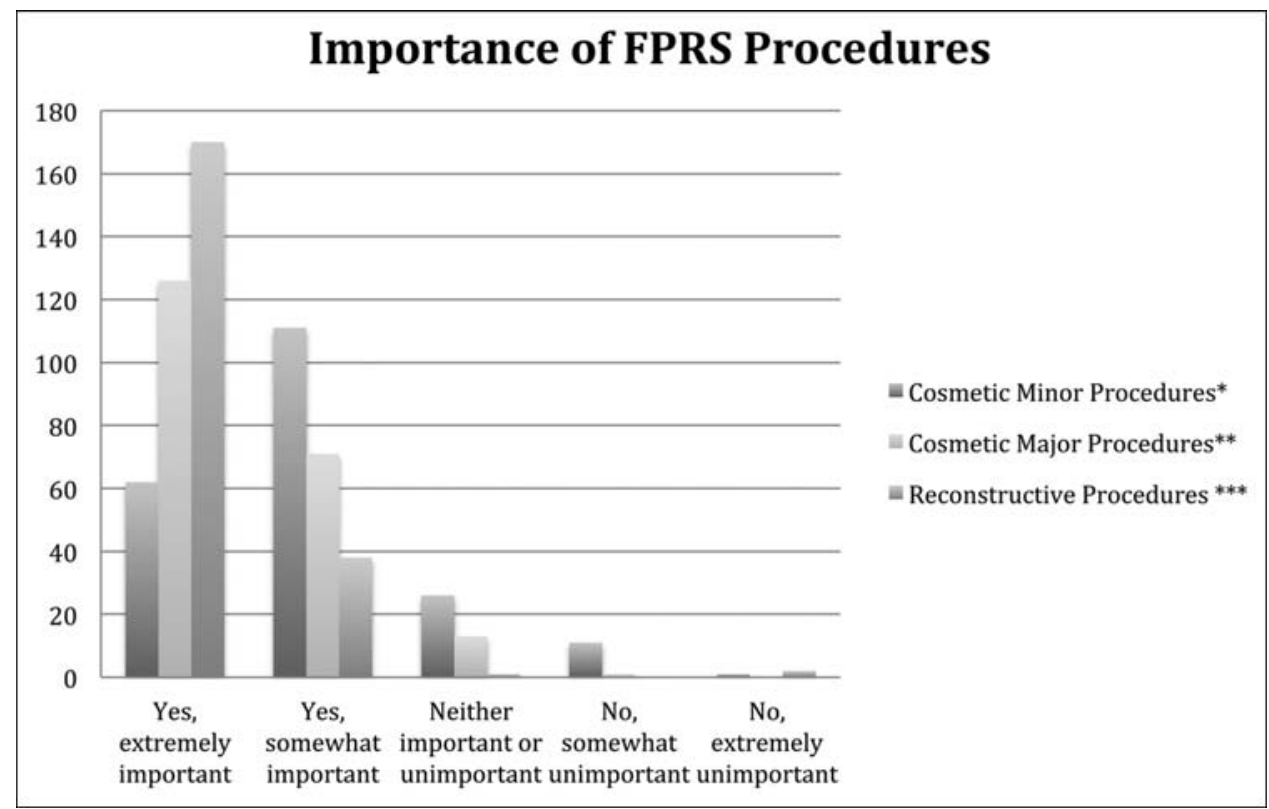

Fig. 2 Importance of Cosmetic Procedures. *Minor procedures = (injections, lasers, chemical peels, etc). ${ }^{* *}$ Major procedures $=($ facelift, rhinoplasty, blepharoplasty, etc.). ${ }^{* * *}$ Reconstructive procedures $=$ (MOHS reconstruction, skin cancer excision, etc). 


\section{Discussion}

Our survey demonstrates that most residents (>98\%) find that FPRS education is important to otolaryngology residency training. The majority of these residents have exposure to cosmetic major and minor procedures while in training (93 and 78\%, respectively); however, only $57 \%$ are able to participate in minor and $81 \%$ in major procedures. This is not surprising for cosmetic procedures, as many of these procedures are performed on a cash basis and patients are paying for a specific surgeon.

Just over half of the respondents (51\%) feel they could receive improved cosmetic facial plastic surgery training during their residency, which is on par with other otolaryngology subspecialties. ${ }^{9}$ This should be of interest to program directors, especially since general otolaryngologists have interest in providing these procedures in their practice. ${ }^{2-4}$ Education should begin with formal lectures ( $>90 \%$ of residents reported exposure to), but as with any surgical or procedural skill, continue with hands-on training with direct supervision and constructive feedback. From initial consultations to the surgical procedures, FPRS and, in particular, cosmetic surgery is very different from many other areas of general otolaryngology.

Strategies to strengthen the FPRS curriculum have been discussed in the past, but perhaps this area should continue to be examined. Only $20 \%$ of the residents who responded had an opportunity to participate in a cosmetic resident clinic. This is an area that could potentially be targeted by otolaryngology residency programs to help increase resident education. Some pharmaceutical companies will provide residents-in-training with sample products on a quarterly basis. Depending on the rules of each institution, these products can be used to supply a free, or reduced cost, resident-run clinic with staff supervision.

The lack of feeling adequately trained in cosmetic procedures by residents in this survey is further evidence of the importance of FPRS fellowships for those who wish to have a cosmetic surgery practice in the future. This also shows it is important for patients who are interested in cosmetic procedures to seek out physicians with proper training. Otolaryngology residents spend their entire residency operating on or around the head and neck. It is hard to imagine physicians with far less experience operating in this area to feel as competent with the anatomy or surgical steps.

The present study is not without limitations. The survey that was distributed was sent to the residency program coordinators and program directors, who were then asked to forward to their residents. We cannot be certain that all residents received the survey. The survey was sent as an anonymous link and, in theory, this survey could have been taken by anyone with access. There is also no way to stop someone from taking the survey multiple times. There was also a low overall number of respondents, which even though consistent with such studies, can introduce bias. A total of $31 \%$ percent of the respondents noted they were either very or moderately interested in a facial plastic fellowship. This percentage is much larger than the percentage of otolaryngology residents that apply to FPRS fellowships each year, furthering concern for selection bias. Finally, although the survey had a good mixture of respondents from all postgraduate year (PGY) levels, junior residents may not be able to answer some questions as accurately when compared with senior residents. Despite these limitations, the present study can serve as an introduction to the disparity in FPRS training perceived amongst otolaryngology residents.

\section{Conclusion}

Facial plastic and reconstructive surgery education continues to be an important aspect of otolaryngology training. The respondents seem to agree with this; however, the exposure to these procedures among residency programs varies. Further, many otolaryngology residents feel their cosmetic facial plastic surgery training could be improved, which is an area to focus on with regards to otolaryngology education.

\section{Location}

The research for this manuscript was conducted at the University of Mississippi Medical Center in Jackson, Mississippi.

Conflict of Interests

The authors have no conflict of interests to declare.

\section{References}

1 Barr JS, Sinno S, Cimino M, Saadeh PB. Clinicians performing cosmetic surgery in the community: a nationwide analysis of physician certification. Plast Reconstr Surg 2015;135(01):92e-98e

2 Burns P, Miller I, Timon C, Walsh M. Otorhinolaryngologists' interest in facial plastic surgery: a survey in the United Kingdom and Ireland. J Laryngol Otol 2008;122(03):299-302

3 Adamson PA, Rubin AM. The otolaryngologist's attitude to facial plastic surgery. J Otolaryngol 1986;15(03):196-200

4 van Pinxteren SAT, Lohuis PJFM, Ingels KJAO, Nolst Trenité GJ. Interest in facial plastic and reconstructive surgery among otorhinolaryngologists: a survey in The Netherlands. Arch Facial Plast Surg 2005;7(02):138-142

5 Miller RH. Otolaryngology residency and fellowship training. The resident's perspective. Arch Otolaryngol Head Neck Surg 1994; 120(10):1057-1061

6 Thomas JR, Graboyes JH. A specific curriculum in facial plastic surgery. Effect on residency training. Arch Otolaryngol Head Neck Surg 1986;112(01):70-72

7 Thomas JR, Ehlert TK, Fenwick J. Facial plastic surgery in the otolaryngology training program: an update. Am J Otolaryngol 1990;11(03):188-190

8 Rhee JS. The JAMA Facial Plastic Surgery Clinical Education Blueprint. JAMA Facial Plast Surg 2015;17(03):167-168

9 Hu A, Sardesai MG, Merati AL, Fung K. Trainee perceptions of laryngology in otolaryngology residency programs. J Otolaryngol Head Neck Surg 2012;41(06):419-425 